\title{
Who benefits from presurgical endoscopic diagnosis: the patient or the surgeon?
}

\section{다 (1) $\odot$}

\author{
Authors \\ Cesare Hassan ${ }^{1}$, Tom Eelbode ${ }^{2}$, Raf Bisschops ${ }^{2}$ \\ Institutions \\ 1 Endoscopy Unit, Nuovo Regina Margherita Hospital, \\ Rome, Italy Department of \\ 2 Gastroenterology, University Hospitals Leuven, Leuven, \\ Belgium \\ Bibliography \\ DOI https://doi.org/10.1055/a-1006-2737 | \\ Endoscopy International Open 2019; 07: E1540-E1541
}

Any patient in the same clinical condition would expect to be treated in the same way, with the same resources and the same competence in order to achieve the best possible outcome. Based on these theoretical scenarios, evidence-based standards are generally dictated by scientific societies as clinical or technical recommendations. Unfortunately, the real world is exactly the opposite, as every patient requires an individualized decision based on concomitant diseases or therapies, different values and preferences, and availability of technical and human resources. This affects the generalizability of available standards, undermining compliance with current recommendations in clinical practice.

When overlooking well-defined standard, the burden of any decision-making process relies on the single professional. When such decisions are associated with substantial risks of morbidity and mortality, some reluctance by professionals may be expected, as it would be they who would be be blamed for any major adverse event. Far from being justified, these personal-when not emotional-reactions generate an irrational excess of diagnostic procedures in an attempt to nullify rather than reasonably decrease any marginal risk. These over-cautious behaviors are ominously known as "defensive" medicine, sadly being reported throughout all the medical and surgical fields. In gastroenterology/endoscopy, this frequently results in an excess of post-endoscopic surveillance, as widely reported in post-polypectomy or Barrett settings. Disappointingly, patients tend to appreciate such an over-zealous approach, triggering a vicious circle between the two main actors of the medical choice.

The main danger in "defensive" medicine is represented by the change in perspective between professionals and patients rather than by the mere waste of economic or financial resour- ces. The main benefit of any "defensive" approach accrues to physicians rather than to their patients. In the unrealistic attempt to acquire the full certainty of his or her choice, physicians transfer what should have been their responsibility onto the patients themselves, exposing them to the risks of unnecessary procedures. It could be argued that this is untrue as patients could also get benefit from such obsessive approach. Isn't it a persuasive belief among patients that an extra exam is better than one fewer?

Of course, not! In this issue of ElO, Sonnenberg A et al. show the catastrophic consequences to patient outcome of the "defensive" approach. In both cases, it was the surgeon who inappropriately over-used endoscopic resources to have the highest possible degree of certainty about the correctness of the surgical indication before operating on the patient. What the authors clearly showed - and patients should understand - is that a "defensive" approach increases the complexity of - rather than simplifying - the clinical choice. The more tests you ask the more cumbersome the process becomes for somewhat unpredictable reasons, such as waiting list, need to stop concomitant medications, and lack of adequate resources. In addition, it should be the same professionals who timely analyze all of this information in order to save precious time. However, when dealing with over-busy professionals - such as surgeons or other interventional figures - what happens is that some of them may transfer responsibility for the ultimate choice to another professional, who may, for example, miss the complete clinical picture or may not be trained enough to deal with urgent cases.

How to prevent "defensive" medicine? What most physicians miss is that the main reason for which formal recommendations represent the current standard of our profession is not 
the fact that they are evidence-based, but that patient-centered methodology is used to support them. The GRADE methodology implies a careful balance between benefit and risk in any formally defined scenarios. The only purpose of the evidence summarized from the literature is to provide exact estimates for both benefit and risk. Anytime the benefit outweighs the harm (or vice versa) for the patient, a procedure is (or is not) indicated. This probabilistic approach is far from the obsession with nullification of risks in "defensive" medicine, and it is usually based on simple and straightforward clinal approaches. Thus, no additional diagnostic test should be considered when a clear benefit for the patient is offset by only a negligible risk of adverse events. In the emergency or urgent setting, a reasonable estimate of the risk/benefit ratio is generally based on fast and accurate cross-sectional radiological examinations, whereas - with the noticeable exception of gastrointestinal bleeding - the additional contribution of diagnostic endoscopy tends to be negligible and inefficient.

It could be argued, however, that even a systematic application of the risk/benefit ratio may be ineffective in the real world. A case may require multidisciplinary complex knowledge or a level of experience that may be lacking in young trainees. In addition, personal prejudices or emotional reactions by professionals may somewhat distort the actual estimate of benefit and risks, as may fatigue or distractions when dealing with multiple cases at the same time.

A possible aid for complex cases may be represented by use of artificial intelligence in supporting physicians in the decisionmaking process as it is increasingly reported for emergency surgery. There are four main advantages of a machine-learning based approach. First, it can be trained on an amount of patient data that exceeds by several-fold the lifetime experience of surgeons or other professionals, and it is also based on complex features weighed in a non-linear way, which far surpasses the capacity of the human mind. Second, it incorporates a large number of variables in its analysis, such as the functional status of the patient or the anesthesiological risk, updating at each variation the suggested treatment. Third, it is capable of multi-tasking so it can deal with a potentially infinite number of patients at any moment, flagging those requiring immediate treatment to the supported surgeon. Fourth, it is not affected by emotional reactions or by fatigue, always basing the decision on an objective estimate of risks and benefits.

\section{Conclusion}

In conclusion, gastrointestinal surgeons should sometimes continue to perform surgery and endoscopists should be aware of the potential delay of life-saving interventions for diagnostic purposes. Multidisciplinary teams, well-defined and shared protocols, as well as new support by artificial intelligence should be able to orientate the decision-making process in an objective and fair way.

\section{Competing interests}

None 